\title{
Tensor-Centric Warfare III: Combat Dynamics with Delta-Strikes
}

\author{
Vladimir Ivancevic ${ }^{1}$, Peyam Pourbeik ${ }^{2}$, Darryn Reid1 \\ ${ }^{1}$ Joint and Operations Analysis Division, Defence Science \& Technology Group, Adelaide, Australia \\ ${ }^{2}$ Cyber and Electronic Warfare Division, Defence Science \& Technology Group, Adelaide, Australia \\ Email: Vladimir.Ivancevi@@dsto.defence.gov.au,Peyam.Pourbeik@dsto.defence.gov.au, \\ Darryn.Reid@dsto.defence.gov.au
}

How to cite this paper: Ivancevic, V., Pourbeik, P. and Reid, D. (2018) Tensor-Centric Warfare III: Combat Dynamics with Delta-Strikes. Intelligent Control and Automation, 9, 107-122.

https://doi.org/10.4236/ica.2018.94009

Received: September 28, 2018

Accepted: November 2, 2018

Published: November 5, 2018

Copyright () 2018 by authors and Scientific Research Publishing Inc.

This work is licensed under the Creative

Commons Attribution International

License (CC BY 4.0).

http://creativecommons.org/licenses/by/4.0/

\begin{abstract}
This paper is the third part of the complex combat dynamics series, called tensor-centric warfare (for the first two parts, see [1] [2]). In the present paper, we extend the tensor combat model from [1] and [2] to model the dynamics of delta-strikes/missiles, which are temporally confined strong kinetic effects. The scenarios analyzed here include both deterministic and random delta-strikes which mimic single, multiple and continuous-time missile attacks. We also look at the bidirectional random strike as well as the general Hamilton-Langevin dynamics framework and provide an interpretation of the results obtained through simulation.
\end{abstract}

\section{Keywords}

Tensor-Centric Warfare, Combat Dynamics with Delta-Strikes/Missiles

\section{Introduction}

Various aspects of warfare modelling have a long and successful history in Defence Science \& Technology Group, Australia. To get a glimpse of the combat modelling in the previous decade, see [3] [4] [5] [6] [7], and in this decade, see [8]-[15].

In the first two papers of the tensor-centric warfare (TCW) series [1] [2], we have developed combat tensor dynamics (generalized from the Lanchester-type equations) with included commutators (for analysis of warfare symmetry) and entropic Lie-dragging (for modeling warfare uncertainty), given by the following tensor equations: 


$$
\begin{aligned}
& \text { Red : } \dot{R}^{a}=k A_{b}^{a} B^{b}+k_{b} F_{c d}^{a b} B^{c} R^{d}+\left[R^{a}, B^{a}\right]+R^{b} \mathfrak{L}_{R} \mathcal{N}_{b}^{a}, \\
& \text { or: } \dot{R}^{a}=\mathcal{R}^{a}, \quad\left(\text { with } \mathcal{R}^{a}=k A_{b}^{a} B^{b}+k_{b} F_{c d}^{a b} B^{c} R^{d}+\left[R^{a}, B^{a}\right]+R^{b} \mathfrak{L}_{R} \mathcal{N}_{b}^{a}\right) \\
& \text { Blue : } \dot{B}^{a}=\kappa C_{b}^{a} R^{b}+\kappa_{b} G_{c d}^{a b} B^{c} R^{d}+\left[B^{a}, R^{a}\right]+B^{b} \mathfrak{L}_{B} \mathfrak{N}_{b}^{a}, \\
& \text { or: } \dot{B}^{a}=\mathcal{B}^{a}, \quad\left(\text { with } \mathcal{B}^{a}=\kappa C_{b}^{a} R^{b}+\kappa_{b} G_{c d}^{a b} B^{c} R^{d}+\left[B^{a}, R^{a}\right]+B^{b} \mathfrak{L}_{B} \mathfrak{N}_{b}^{a}\right),
\end{aligned}
$$

where the Red and Blue forces are defined as vector-fields, $R^{a}=R^{a}(\boldsymbol{x}, t)$ and $B^{a}=B^{a}(\boldsymbol{x}, t)$, and the terms on the right-hand side respectively represent linear Lanchester-type terms, quadratic Lanchester-type terms, commutators and entropic Lie-dragging terms (for detailed explanation see description of Equation (7) in [2]).

In the present paper, we extend this smooth tensor combat dynamics, with various combinations of temporally confined strong kinetic effects, which we call delta-strikes/missiles, developed in a perturbative fashion (adding more-and-more complex terms at each step). To allow for analysis and comparison of the methodology we have adopted a similar scenario framework to the first TCW-paper [1], with added various combinations of delta-strikes/missiles. We formulate this combat strike dynamics around the high-dimensional Dirac delta-function $\delta(t)^{1}$ (see Figure 1). Since the response of any (complex) system to the delta-function input is called the impulse response, in the simulations below we will look at various impulse responses of the Red and Blue forces to the delta-strikes from the opposite side.

The initial scenario consists of nine Red and nine Blue aircraft which can be potential targets and could also act as a missile strike force. This will allow us to analyze the combat dynamics of our delta-strikes. We will initially look at a single strike which will involve a missile attached by a Red entity targeting and striking a single Blue target and after a short delay Blue is responding in the same way.

Then we introduce a multi-strike scenario which will model multiple missile attacks by Red on a Blue target and Blue responding in the same way. The multi-strike scenario is further extended by continuous (rapid) fire by Red on specific Blue targets and Blue responding in the same way. This discrete + continuous delta-strike spectrum is then naturally extended with a bidirectional random strike scenario, modeled and simulated to depict a missile exchange between the two adversaries.

${ }^{1}$ Recall that the Dirac delta-function, $\delta(t)$, in mathematics called delta-distribution and in control engineering called impulse function, represents the limit of the sequence of zero-mean Gaussian distributions:

$$
\delta(t)=\lim _{d \rightarrow 0} \frac{1}{|d| \sqrt{\pi}} \exp \left[-\left(\frac{t}{d}\right)^{2}\right] .
$$

Its main properties are:

$$
\delta(t)=\left\{\begin{array}{ll}
+\infty, & t=0 \\
0, & t \neq 0
\end{array}, \quad \int_{-\infty}^{\infty} \delta(t) \mathrm{d} t=1, \quad \int_{-\infty}^{\infty} f(t) \delta(t) \mathrm{d} t=f(0),(\text { for any } f(t) \in \mathbb{R}) .\right.
$$




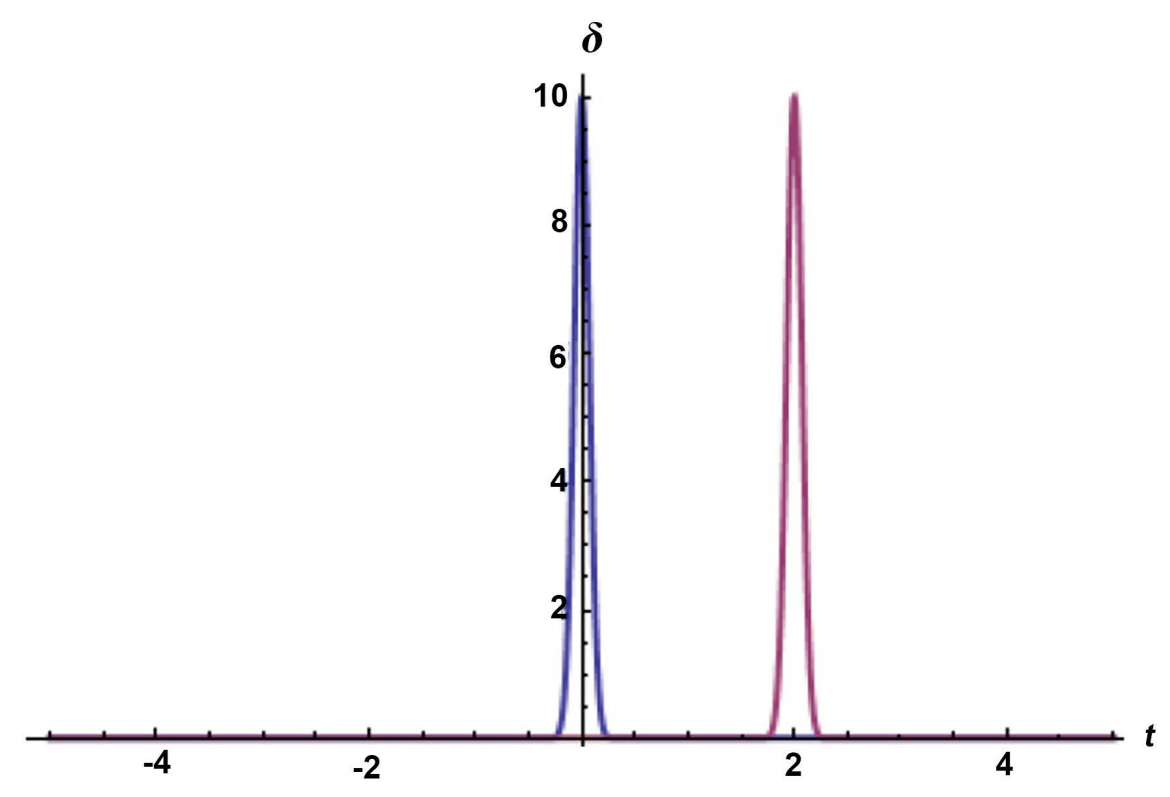

Figure 1. Narrow Gaussian approximation for the shifted Dirac delta-function: $\delta(t-\tau)=10 \exp \left[-100(t-\tau)^{2}\right]$, corresponding to the values of $\tau=0$ (center) and $\tau=2$ (right).

Next, we introduce stochastic forces of arbitrary (non-delta) nature into our model by utilizing a simple Langevin representation of the discrete, continuous and bidirectional delta-strike spectrum between the Red and Blue aircraft. In the final instance we give the formal Hamiltonian formulation of this stochastic Langevin model. This will allow for a probabilistic interpretation of the battlefield and hence allow for a problem solving capability which is much closer to the reality of warfare.

Thus our aim is to develop sophisticated mathematical tools to aid problem formulation and solution rather than facilitate immediate predictive capability with unrealistically simplistic models (see [3] [4] [5] [6] [7], and [8]-[15]).

\section{Combat Dynamics with Delta-Strikes}

In this section we will perturbatively extend tensor Lie-Lanchester Equations (1) so to develop general combat tensor dynamics with both deterministic and random delta-strikes. We formulate this combat $\delta$-strike dynamics in the following seven steps (using Einstein's summation convention over repeated indices), corresponding to the physical background steps given in the Appendix.

Single strike. We start with the pure deterministic framework, with the basic $n \mathrm{D}$ Red $-R^{a}$ against Blue $-B^{a}$ combat dynamics in $\mathbb{R}^{n}$ with a single strike (rocket) on each side:

$$
\begin{aligned}
& \text { Red : } \dot{R}^{a}=\mathcal{R}^{a}-\gamma_{b}^{a} R^{b}+\alpha^{a} \delta\left(t-\tau_{R}\right), \\
& \text { Blue : } \dot{B}^{a}=\mathcal{B}^{a}-\chi_{b}^{a} B^{b}+\beta^{a} \delta\left(t-\tau_{B}\right),
\end{aligned}
$$


where $\gamma_{b}^{a}$ and $\chi_{b}^{a}$ are the Red and Blue "damping" coefficients, respectively, and $\tau_{R}$ and $\tau_{B}$ are the Red and Blue strike times; the terms with $\delta$-functions represent single strikes/missiles.

Multi-strike. Now we generalize $\delta$-functions in Equations (3) to include $N$ multi-strikes for the Red forces (happening at times $\tau_{j}^{R}$ ) and $M$ multi-strikes for the Blue forces (happening at times $\tau_{j}^{B}$ ), representing one-at-a-time (or, discrete time) multi-missile launchers:

$$
\begin{aligned}
& \text { Red : } \dot{R}^{a}=\mathcal{R}^{a}-\gamma_{b}^{a} R^{b}+\sum_{j=1}^{N} \alpha_{j}^{a} \delta\left(t-\tau_{j}^{R}\right), \\
& \text { Blue : } \dot{B}^{a}=\mathcal{B}^{a}-\chi_{b}^{a} B^{b}+\sum_{j=1}^{M} \beta_{j}^{a} \delta\left(t-\tau_{j}^{B}\right) .
\end{aligned}
$$

Discrete and continuous striking spectra. Now we extend Equations (4) by adding continuous striking spectrum, representing many-at-a-time (or, continuous time) rocket launchers on both sides:

$$
\begin{aligned}
& \text { Red : } \dot{R}^{a}=\mathcal{R}^{a}-\gamma_{b}^{a} R^{b}+\sum_{j=1}^{N} \alpha_{j}^{a} \delta\left(t-\tau_{j}^{R}\right)+\int_{t_{0}}^{t_{1}} \alpha^{a}(t) \delta\left(t-\tau_{R}\right) \mathrm{d} t, \\
& \text { Blue : } \dot{B}^{a}=\mathcal{B}^{a}-\chi_{b}^{a} B^{b}+\sum_{j=1}^{M} \beta_{j}^{a} \delta\left(t-\tau_{j}^{B}\right)+\int_{t_{0}}^{t_{1}} \beta^{a}(t) \delta\left(t-\tau_{B}\right) \mathrm{d} t,
\end{aligned}
$$

where $\delta$-terms with sums correspond to discrete-time launchers (one rocket at a time) and $\delta$-terms with integrals correspond to continuous-time launchers (many rockets at a time).

Bidirectional random strikes. Now we extend Equations (5) by including bidirectional $( \pm$ ) random strikes (i.e., random bidirectional striking at each other), with Red- and Blue-related random numbers: $\left(\rho^{R}, \rho^{B}\right) \in[0,1]$ :

$$
\begin{aligned}
\text { Red : } \dot{R}^{a}= & \mathcal{R}^{a}-\gamma_{b}^{a} R^{b}+\sum_{j=1}^{N} \alpha_{j}^{a} \delta\left(t-\tau_{j}^{R}\right)+\int_{t_{0}}^{t_{1}} \alpha^{a}(t) \delta\left(t-\tau_{R}\right) \mathrm{d} t \\
& +\alpha^{a} \sum_{j=1}^{N} \delta\left(t-\tau_{j}^{R}\right)\left( \pm \rho^{R}\right)_{j}, \\
\text { Blue }: \dot{B}^{a}= & \mathcal{B}^{a}-\chi_{b}^{a} B^{b}+\sum_{j=1}^{M} \beta_{j}^{a} \delta\left(t-\tau_{j}^{B}\right)+\int_{t_{0}}^{t_{1}} \beta^{a}(t) \delta\left(t-\tau_{B}\right) \mathrm{d} t \\
& +\beta^{a} \sum_{j=1}^{M} \delta\left(t-\tau_{j}^{B}\right)\left( \pm \rho^{B}\right)_{j} .
\end{aligned}
$$

Simple Langevin equations. Now we move to the simple stochastic Langevin dynamics framework (see [16] [17] [18] [19]) and extend Equations (6) with random (non-delta) forces Red- $f_{\text {rnd }}^{a}(t)$ and Blue- $g_{\text {rnd }}^{a}(t)$ :

$$
\begin{aligned}
\text { Red }: \dot{R}^{a}= & \mathcal{R}^{a}-\gamma_{b}^{a} R^{b}+\sum_{j=1}^{N} \alpha_{j}^{a} \delta\left(t-\tau_{j}^{R}\right)+\int_{t_{0}}^{t_{1}} \alpha^{a}\left(\tau_{R}\right) \delta\left(t-\tau_{R}\right) \mathrm{d} t \\
& +\alpha^{a} \sum_{j=1}^{N} \delta\left(t-\tau_{j}^{R}\right)\left( \pm \rho^{R}\right)_{j}+f_{\text {rnd }}^{a}(t), \\
\text { Blue }: \dot{B}^{a}= & \mathcal{B}^{a}-\chi_{b}^{a} B^{b}+\sum_{j=1}^{M} \beta_{j}^{a} \delta\left(t-\tau_{j}^{B}\right)+\int_{t_{0}}^{t_{1}} \beta^{a}\left(\tau_{B}\right) \delta\left(t-\tau_{B}\right) \mathrm{d} t \\
& +\beta^{a} \sum_{j=1}^{M} \delta\left(t-\tau_{j}^{B}\right)\left( \pm \rho^{B}\right)_{j}+g_{\text {rnd }}^{a}(t) .
\end{aligned}
$$


Hamilton-Langevin dynamics. Finally, we formulate the general Langevin dynamics in the Hamiltonian (symplectic geometric) framework to be able to extend the simple random forces Red- $f_{\text {rnd }}^{a}(t)$ and Blue- $g_{\text {rnd }}^{a}(t)$ in Equations (6) with the general Langevin forces derived from the hypothetical global Red-Blue combat Hamiltonian energy function, $H\left(R^{a}, B^{a}\right)$, defined in an Ising-Hamiltonian fashion as:

$$
H=-J_{a b} R^{a} B^{b}=-\ln \left[p_{0}\left(R^{a}, B^{a}\right)\right],
$$

where $J_{a b}$ is the Red-Blue interaction tensor, defined as the combined Red-Blue Combat tensor (see [1]): $J_{a b}=A_{b}^{a} C_{a}^{b} \rho_{a b}$, where $\rho_{a b}=\rho \delta_{a b}$ is the random identity matrix (or, random Kronecker delta), and $p_{0}\left(R^{a}, B^{a}\right)$ is the equilibrium probability distribution of the coupled $\left(R^{a}, B^{a}\right)$-system. Besides, instead of the separate Poisson brackets $k_{B} T\left[R^{a}, R^{b}\right]$ for the Red forces and $k_{B} T\left[B^{a}, B^{b}\right]$ for the Blue forces (with the Boltzmann and temperature constants), we will replace them with the coupled Red-Blue Poisson bracket $\left[R^{a}, B^{a}\right]$, which is the Lie bracket commutator (from Equations (1)): $\left[R^{a}, B^{a}\right]=[$ Red, Blue $]=\mathfrak{L}_{R} B^{a}=-\mathfrak{L}_{B} R^{a}$, where $\mathfrak{L}_{R}$ and $\mathfrak{L}_{B}$ denote the Lie derivatives in the direction of the Red and Blue vector-fields, respectively. In this way, we come to our final Red-Blue combat model in the form of tensor Hamilton-Langevin equations:

$$
\begin{aligned}
\text { Red : } \dot{R}^{a}= & \mathcal{R}^{a}-\gamma_{b}^{a} R^{b}+\sum_{j=1}^{N} \alpha_{j}^{a} \delta\left(t-\tau_{j}^{R}\right)+\int_{t_{0}}^{t_{1}} \alpha^{a}(t) \delta\left(t-\tau_{R}\right) \mathrm{d} t \\
& +\alpha^{a} \sum_{j=1}^{N} \delta\left(t-\tau_{j}^{R}\right)\left( \pm \rho^{R}\right)_{j}+\left[R^{a}, B^{a}\right] \frac{\partial H}{\partial B^{b}} R^{b}-\gamma^{a b} \frac{\partial H}{\partial B^{b}}+f_{\text {rnd }}^{a}(t), \\
\text { Blue }: \dot{B}^{a}= & \mathcal{B}^{a}-\chi_{b}^{a} B^{b}+\sum_{j=1}^{M} \beta_{j}^{a} \delta\left(t-\tau_{j}^{B}\right)+\int_{t_{0}}^{t_{1}} \beta^{a}(t) \delta\left(t-\tau_{B}\right) \mathrm{d} t \\
& +\beta^{a} \sum_{j=1}^{M} \delta\left(t-\tau_{j}^{B}\right)\left( \pm \rho^{B}\right)_{j}+\left[B^{a}, R^{a}\right] \frac{\partial H}{\partial R^{b}} B^{b}-\chi^{a b} \frac{\partial H}{\partial R^{b}}+g_{\text {rnd }}^{a}(t) .
\end{aligned}
$$

This is our final tensor Lie-Lanchester combat model with $\delta$-strikes/missiles.

For completeness purpose, we finish this section with two probabilistic interpretations (compare with [16] [17] [18] [19]) of the random parts of the Hamilton-Langevin Equations (7):

- Fokker-Planck equations:

$$
\begin{aligned}
& \text { Red }: \frac{\partial P(R, t)}{\partial t}=\frac{\partial}{\partial R^{a}}\left(-\left[R^{a}, B^{a}\right] \frac{\partial H}{\partial B^{b}} R^{b}+\gamma^{a b} \frac{\partial H}{\partial B^{b}}\right) P(R, t), \\
& \text { Blue }: \frac{\partial P(B, t)}{\partial t}=\frac{\partial}{\partial B^{a}}\left(-\left[B^{a}, R^{a}\right] \frac{\partial H}{\partial R^{b}} B^{b}+\chi^{a b} \frac{\partial H}{\partial R^{b}}\right) P(B, t),
\end{aligned}
$$

with the Red and Blue stationary solutions given by the equilibrium distributions: $P(R, t)=c_{R} \mathrm{e}^{-H}$ and $P(B, t)=c_{B} \mathrm{e}^{-H}$ with constants $\left(c_{R}, c_{B}\right)$; and

- Feynman path integrals:

$\operatorname{Red}: \int \mathrm{d} R \mathrm{~d} \tilde{R} P(R, \tilde{R})=N \int \mathrm{d} R \mathrm{~d} \tilde{R} \exp [L(R, \tilde{R})]$, with

$$
L(R, \tilde{R})=\int \mathrm{d} t\left[R^{a} \gamma_{a b} R^{b}-\tilde{R}^{a}\left(\delta_{a b} \dot{R}^{b}-\left[R^{a}, B^{a}\right] \frac{\partial H}{\partial B^{b}} R^{b}+\gamma^{a b} \frac{\partial H}{\partial B^{b}}\right)\right]
$$


Blue : $\int \mathrm{d} B \mathrm{~d} \tilde{B} P(B, \tilde{B})=M \int \mathrm{d} B \mathrm{~d} \tilde{B} \exp [L(B, \tilde{B})]$, with

$$
L(B, \tilde{B})=\int \mathrm{d} t\left[B^{a} \gamma_{a b} B^{b}-\tilde{B}^{a}\left(\delta_{a b} \dot{B}^{b}-\left[B^{a}, R^{a}\right] \frac{\partial H}{\partial R^{b}} B^{b}+\chi^{a b} \frac{\partial H}{\partial R^{b}}\right)\right]
$$

where $\left(\tilde{R}^{a}, \tilde{B}^{a}\right)$ represent the (Red, Blue) auxiliary-response variables and $(N, M)$ are their respective normalization constants.

\section{Simulations and Discussion}

Simulations of the combat strike-dynamics are performed in Mathematica ${ }^{@}$ as follows. For the calibration/comparing purpose, we firstly show the simulation of the tensor Lie-Lanchester Equations (1) given in Figure 2. Also, for better simulation results, we have approximated the Dirac $\delta$-function by the narrow Gaussian (see Figure 1) implemented in Mathematica as: delta $\left[t_{-}, \tau_{-}\right]:=10 \operatorname{Exp}\left[-100(t-\tau)^{2}\right]$. We perform the complex impulse-response simulations as follows:

1) We start with the single strike combat simulation (see Figure 3), given by Equations (3), with the following parameters: $\tau_{R}=0.4 t_{1}, \tau_{B}=0.6 t_{1}, \alpha=0.5 \rho_{1}$, $\beta=0.5 \rho_{2}, \quad \gamma_{b}^{a}=\gamma=0.5 \rho_{3}, \quad \chi_{b}^{a}=\chi=0.5 \rho_{4}$, where $t_{1}=t$ Fin is the final simulation time (= 10 units), $\left(\rho_{1}, \rho_{2}, \rho_{3}, \rho_{4}\right)$ are real pseudo-random numbers generated from the $[0,1]$-interval.

2) Next, we simulate the multi-strike combat (see Figure 4), given by Equations (4), with the following new parameters: $N N=15 ; M M=13$;

3) Then, we simulate both discrete and continuous strike-spectra (see Figure 5), given by Equations (5).

4) Then, we simulate bidirectional random strikes (see Figure 6), given by Equations (6).

5) As a final simulation (see Figure 7), we have implemented the Hamilton-Langevin Equations (8), where we have simplified $\gamma$ and $\chi$ tensors and both skipped the computation-heavy Lie bracket terms (used in [2] for determining the warfare symmetry) and additional random forces, since all implemented components already include random numbers.
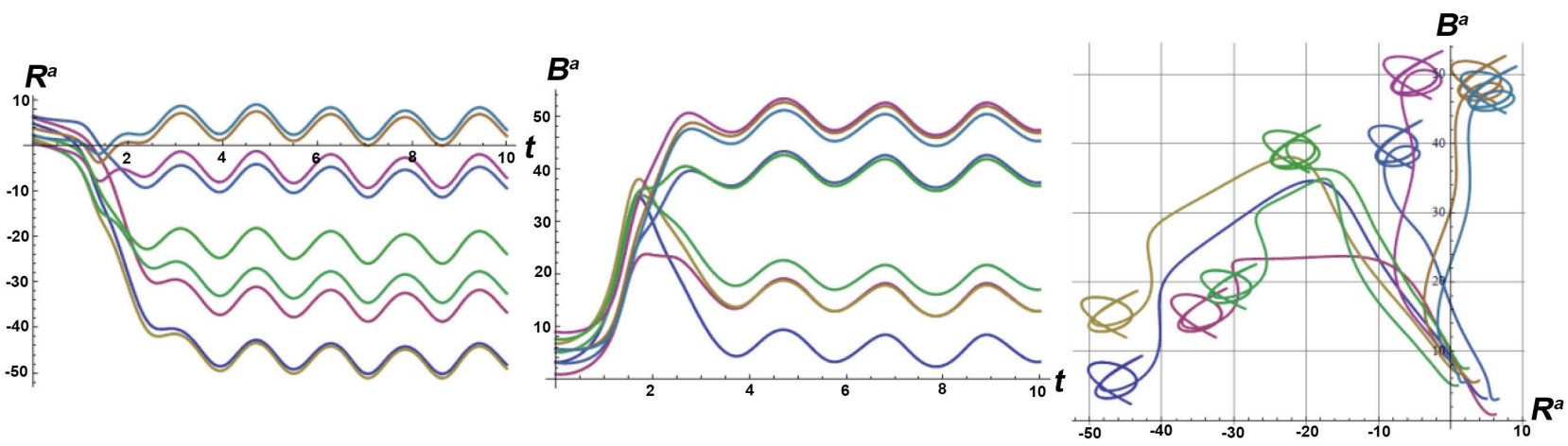

Figure 2. Sample simulation of the combat tensor Lie-Lanchester Equations (1)—for the comparison/calibration purpose: Red amplitude (left) is losing, Blue amplitude (center) is winning and Red-Blue phase plot (right) shows nine points of conflict; the amplitudes range from -50 to 50 . 


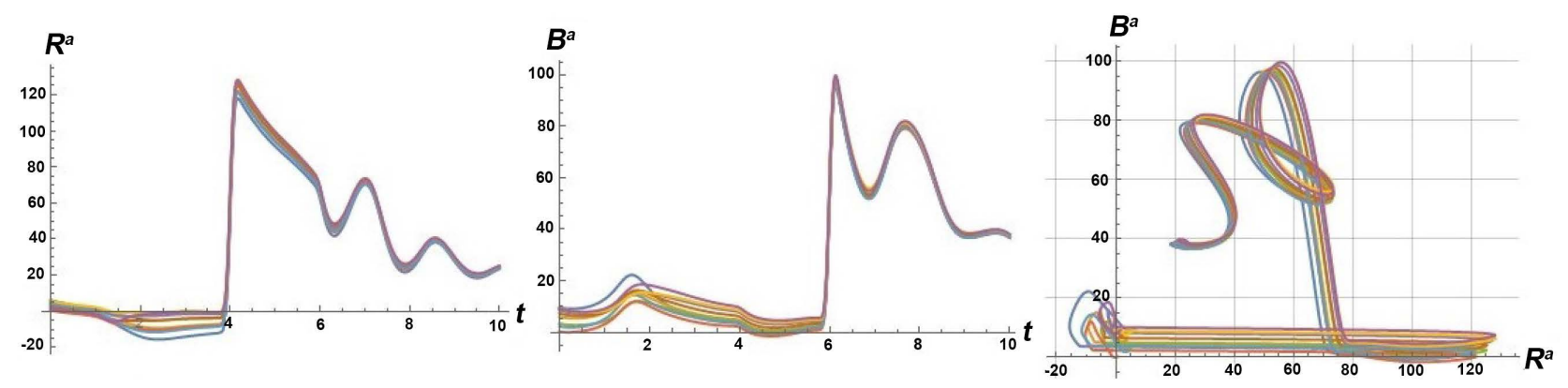

Figure 3. Sample Red-Blue combat simulation for 10 time units of the single strike Equations (3) with the parameters given in the text. We can see that the Red (attacker) strike comes first (left) and the Blue (defender) responds later (center). The amplitudes range up to 150 . The Red-Blue phase-plot (right) already shows complex combat dynamics.
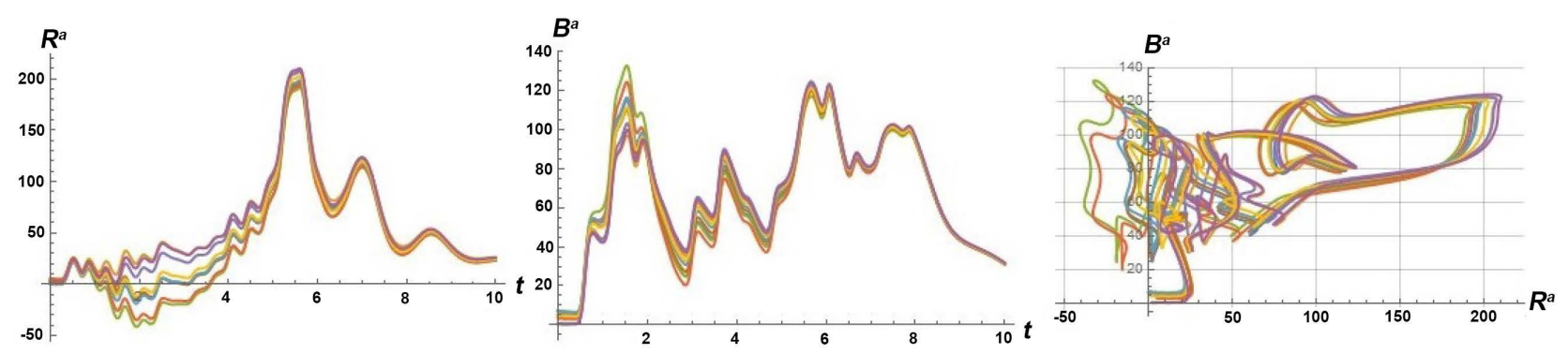

Figure 4. Sample Red-Blue combat simulation for 10 time units of the multi-strike Equations (4) with the parameters given in the text. Here the amplitudes are several times higher than in the single strike, for the Red (left) the amplitude is all positive and for the Blue (center) it starts negative and then grows positive. The Red-Blue phase-plot (right) is even more complex.
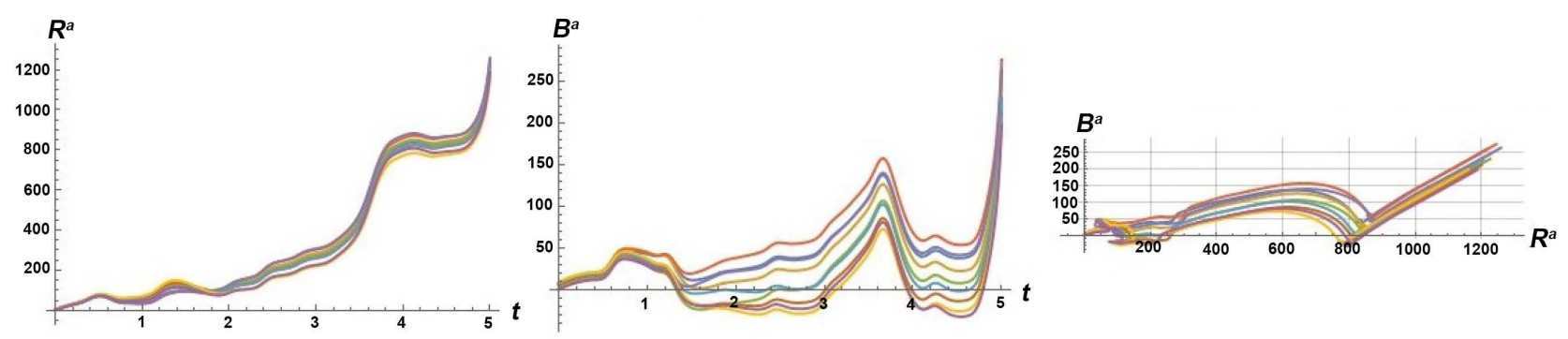

Figure 5. Sample Red-Blue combat simulation for 5 time units with both discrete and continuous strike-spectra given by Equations. (5) with the parameters given in the text. The amplitudes are similar as before, with the roles reversed: the Blue (center) the amplitude is all positive and for the Red (left) it starts negative and then grows positive. The Red-Blue phase-plot (right) is somewhat less complex, showing the filtering capacity of the integral in the continuous spectrum.
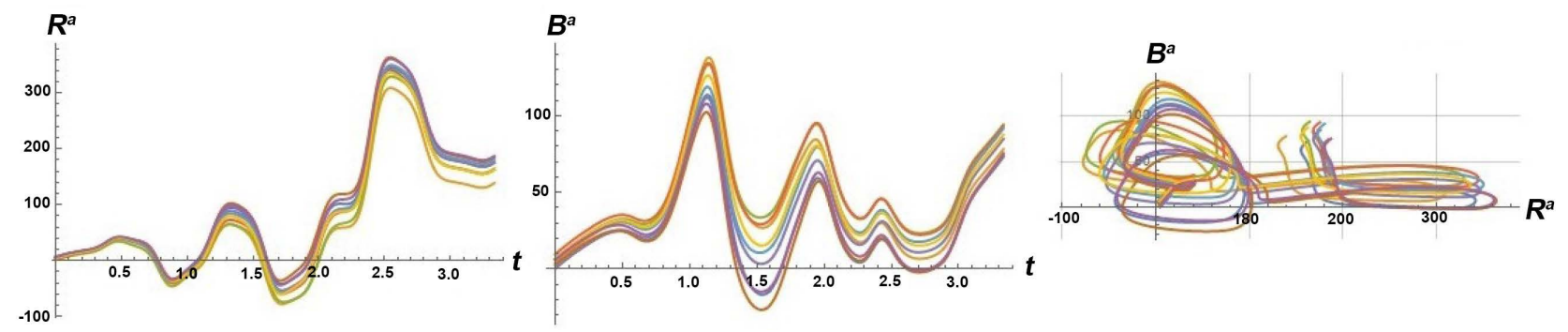

Figure 6. Sample Red-Blue combat simulation for 3.3 time units with added bidirectional random strikes given by Eqs. (6) with the parameters given in the text. Since complexity increases, we had to shorten simulation time. A characteristic signature is shown in the Red-Blue phase plane. 


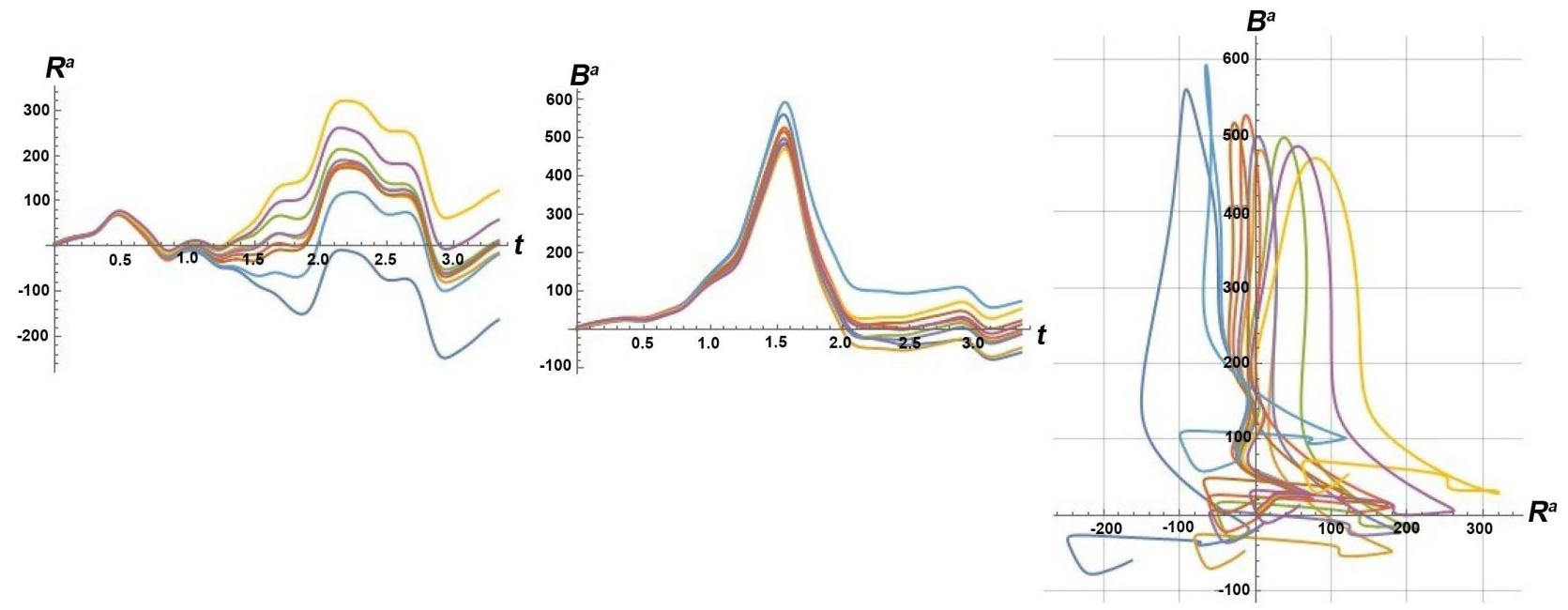

Figure 7. Sample Red-Blue combat simulation for 3.3 time units with added Hamilton-Langevin strikes given by Equations (8) with the parameters given in the text. Here we have even higher complexity with a new signature in the Red-Blue phase plane.

Now, we discuss various simulated scenarios with delta-strikes as follows:

Single strike. The initial scenario consisted of nine Red and nine Blue aircraft which were both potential targets and missile strike forces. We have started with a single strike from each side, $\alpha^{a} \delta\left(t-\tau_{R}\right)$ on the Red side, and $\beta^{a} \delta\left(t-\tau_{B}\right)$ the Blue side, which involved a missile attached by a Red entity targeting and striking a single Blue target and after a short delay Blue was responding in the same way. We can see from Figure 3 that since the Red (attacker) strike comes first and the Blue (defender) responds later, the Red-Blue phase-plot already shows quite different and more complex dynamics than the smooth dynamics on the initial Figure 2. From control-theoretic perspective, in the time plots of the Red and Blue forces we can see the expected effect of the impulse control input followed by the transients. The effect of the single impulse on each side is the convergence of the trajectories in the phase plane, forming the first "combat signature" for this specific type of attack.

Multi-strike. Next, we have introduced a discrete-time multi-missile attacks by Red, $\sum_{j=1}^{N} \alpha_{j}^{a} \delta\left(t-\tau_{j}^{R}\right)$, on a Blue target and Blue, $\sum_{j=1}^{M} \beta_{j}^{a} \delta\left(t-\tau_{j}^{B}\right)$, responding in the same way. We can see from Figure 4 that the amplitudes are now several times higher than in the single strike, for the Red the amplitude is all positive and for the Blue it starts negative and then grows positive. In contrast to the previous Figure, we can see now that the sequence of impulses leads to general irregularity, determined by the rate of the sequence of strikes, which cumulatively results in the increased complexity of the combat signature in the Red-Blue phase-plot.

Discrete and continuous striking spectra. The above multi-strike scenario has been further extended by continuous-time fire by Red, $\int_{t_{0}}^{t_{1}} \alpha^{a}(t) \delta\left(t-\tau_{R}\right) \mathrm{d} t$, on specific Blue targets and Blue, $\int_{t_{0}}^{t_{1}} \beta^{a}(t) \delta\left(t-\tau_{B}\right) \mathrm{d} t$, responding in the same way. We can see from Figure 5 that the continuous spectrum defined by the integral of the high-dimensional impulse function has the "low-pass filtering" effect, which drastically reduces the overall complexity. This is related to the global 
resonance of the Red-Blue combat system. In other words, tuning the time intervals between the strikes produces the resonance effect (like in the radio tuning to a station), giving the significant increase in the amplitudes for both forces-in a half time (five units) the amplitudes are about six times bigger. However, this reduction of the overall complexity has the highly-potent psychological effect, as was well-known as "Katyusha-effect" in the WW2: the continuous sound and vibration reduces morale and effects the combat readiness of the soldiers and commanders.

Bidirectional random strikes. The above discrete + continuous delta-strike spectrum has been naturally extended with a bidirectional random strike scenario, $\alpha^{a} \sum_{j=1}^{N} \delta\left(t-\tau_{j}^{R}\right)\left( \pm \rho^{R}\right)_{j}$ by the Red forces and $\beta^{a} \sum_{j=1}^{M} \delta\left(t-\tau_{j}^{B}\right)\left( \pm \rho^{B}\right)_{j}$ by the Blue forces, simulated to depict a missile exchange between the two adversaries. We can see from Figure 6 that the overall combat complexity again increases but with obvious filtering effect from the continuous fire (many at a time). The combat signature in the Red-Blue phase-plot reappears, but is much smoother (low-pass filtering effect is still present).

Hamilton-Langevin dynamics. In the final instance we have given the formal Hamilton-Langevin stochastic generalization of the previous models. This stochastic formulation allows for a probabilistic interpretation of the battlefield and hence allows for a problem solving capability which is much closer to the reality of warfare. We can see from Figure 7 that adding the Hamilton-Langevin stochastic (non-delta) forces to the combat scenario from the previous Figure again adds the complexity to the combat signature in the Red-Blue phase-plane. Basically, the more noise is added to dynamics, the dynamics becomes more complex. Introduction of random fluctuations into a complex dynamical/control system like our Red-Blue combat system, where the parts of the overall system are interacting (Red and Blue are engaging each other), results in the significant increase of the overall complexity/nonlinearity. However, we can see from the combat signature that the overall Red-Blue combat system is still performing within the edge of chaos (but not over the edge), where it is supposed to perform at its best (in the AI commutinty, it is generally assumed that neural networks have their highest performance at the edge of chaos, see [20] and the references therein).

Complete TCW equation. (with transparent terms) read:

$$
\begin{gathered}
{\stackrel{\dot{R}^{a}}{\text { Rediecfield }}}^{\text {lin.Lanchaster }}=\overbrace{k A_{b}^{a} B^{b}}^{\text {quad.Lanchaster }}+\overbrace{k_{b} F_{c d}^{a b} R^{c} B^{d}}^{\text {que.dragging }}+\overbrace{R^{b} \mathcal{L}_{R} N_{b}^{a}}^{\text {Lis. }}+\overbrace{\left[R^{a}, B^{a}\right]}^{\text {war.symmetry }}+\overbrace{\delta R(\mathrm{H}-\mathrm{L})^{a}}^{\text {delta.strikes }}, \\
\overbrace{\dot{B}^{a}}^{\text {Blue.vecfield }}=\overbrace{\kappa C_{b}^{a} R^{b}}^{\text {lin.Lanchaster }}+\overbrace{\kappa_{b} G_{c d}^{a b} R^{c} B^{d}}^{\text {quad.Lanchaster }}+\overbrace{B^{b} \mathcal{L}_{B} M_{b}^{a}}^{\text {Lie.dragging }}+\overbrace{\left[B^{a}, R^{a}\right]}^{\text {war.symmetry }}+\overbrace{\delta B(\mathrm{H}-\mathrm{L})^{a}}^{\text {delta.strikes }},
\end{gathered}
$$

where Hamilton-Langevin delta strikes, $\delta R(\mathrm{H}-\mathrm{L})^{a}$ and $\delta B(\mathrm{H}-\mathrm{L})^{a}$, read:

$$
\begin{aligned}
\delta R(\mathrm{H}-\mathrm{L})^{a}= & \overbrace{\sum_{j=1}^{N} \alpha_{j}^{a} \delta\left(t-\tau_{j}^{R}\right)}^{\text {disc.spectrum }}+\overbrace{\int_{t_{0}}^{t_{1}} \alpha^{a}(t) \delta\left(t-\tau_{R}\right) \mathrm{d} t}^{\text {cont.spectrum }}+\overbrace{\alpha^{a} \sum_{j=1}^{N} \delta\left(t-\tau_{j}^{R}\right)\left( \pm \rho^{R}\right)}^{\text {bidirect.rnd }} \\
& +\overbrace{\left[R^{a}, B^{a}\right] \frac{\partial H}{\partial B^{b}}}^{\text {RedHam.vecfield }}-\overbrace{\gamma_{b}^{a} R^{b}}^{\text {self.dissipat }}-\overbrace{\gamma_{b}^{a} \frac{\partial H}{\partial B^{b}}}^{\text {oppon.dissipat }}+\overbrace{f_{\text {rnd }}^{a}(t),}^{\text {rnd.force }},
\end{aligned}
$$




$$
\begin{aligned}
\delta B(\mathrm{H}-\mathrm{L})^{a}= & \overbrace{\sum_{j=1}^{M} \beta_{j}^{a} \delta\left(t-\tau_{j}^{B}\right)}^{\text {disc.spectrum }}+\overbrace{\int_{t_{0}}^{t_{1}} \beta^{a}(t) \delta\left(t-\tau_{B}\right) \mathrm{d} t}^{\text {cont.spectrum }}+\overbrace{\beta^{a} \sum_{j=1}^{M} \delta\left(t-\tau_{j}^{B}\right)\left( \pm \rho^{B}\right)}^{\text {bidirect.rnd }} \\
& +\overbrace{\left[B^{a}, R^{a}\right] \frac{\partial H}{\partial R^{b}}}^{\text {BlueHam.vecfield }}-\overbrace{\chi_{b}^{a} B^{b}}^{\text {self.dissipat }}-\overbrace{\chi_{b}^{a} \frac{\partial H}{\partial R^{b}}}^{\text {oppon.dissipat }}+\overbrace{g_{\text {rnd }}^{a}(t)}^{\text {rnd.force }} .
\end{aligned}
$$

\section{Conclusions and Future Work}

The purpose of this paper, the third part of the tensor-centric warfare (TCW) series, was to introduce a new framework for modeling complex warfighting $(\mathrm{MCW})$, by combining continuous tensor quantities with temporally confined strong kinetic effects, which we call delta-strikes/missiles. The verification and/or validation of the proposed framework against the real military examples will be the subject of the future study.

We have presented various combat Red-Blue dynamics of delta-strikes, both deterministic and random-together with the corresponding impulse responses on the opposite side. We have shown that the TCW methodology presented in our previous papers [1] and [2] can be applied to analyzing the dynamic behavior of both deterministic and random delta-strike which mimic single, multiple and continuous-time missile attacks.

Also, Mathematica simulation of the Red-Blue pair of Fokker-Planck equations and the corresponding Red-Blue pair of Feynman path integrals is planned for the future study. Also, we foresee further development of the TCW framework to include tensors for learning and adaptations using deep learning. This will also involve developing the "Entropy Battle" software environment as an experimental testbed for future TCW development. The Entropy Battle will form the backbone of a future Battle Simulation Environment which is hoped to be utilized within the context of military planning.

\section{Acknowledgements}

The authors are grateful to Dr. Tim McKay and Dr. Brandon Pincombe, Joint and Operations Analysis Division, Defence Science \& Technology Group, Australia-for their constructive comments which have improved the quality of this paper.

\section{Conflicts of Interest}

The authors declare no conflicts of interest regarding the publication of this paper.

\section{References}

[1] Ivancevic, V., Pourbeik, P. and Reid, D. (2018) Tensor-Centric Warfare I: Tensor Lanchester Equations. Intelligent Control and Automation, 9, 11-29. https://doi.org/10.4236/ica.2018.92002

[2] Ivancevic, V., Reid, D. and Pourbeik, P. (2018) Tensor-Centric Warfare II: Entropic Uncertainty Modeling. Intelligent Control and Automation, 9, 30-51. 
https://doi.org/10.4236/ica.2018.92003

[3] Pincombe, A.H. and Pincombe, B.M. (2003) A Markov Based Method for Military Analysis. Australian Society for Operations Research Bulletin, 22, 2-8,

[4] Pincombe, A.H. and Pincombe, B.M. (2007) Markov Modelling on the Effectiveness of Sanctions: A Case Study of the Falklands War. ANZIAM Journal, 48, 527-541. https://doi.org/10.21914/anziamj.v48i0.80

[5] Pincombe, A.H. and Pincombe, B.M. (2008) Tractable Approximations to Multistage Decisions in Air Defence Scenarios. ANZIAM Journal, 49, 273-288. https://doi.org/10.21914/anziamj.v49i0.349

[6] Pincombe, A.H., Pincombe, B.M. and Pearce, C.E.M. (2009) Putting the Art before the Force. ANZIAM Journal, 49, 482-496.

[7] Pincombe, A.H., Pincombe, B.M. and Pearce, C.E.M. (2009) A Simple Battle Model with Explanatory Power. ANZIAM Journal, 49, 497-511.

[8] Kosowski, L.R., Pincombe, A.H. and Pincombe, B.M. (2010) Irrelevance of the Fractal Dimension Term in the Fractal Attrition Equation, ANZIAM Journal, 52, 988-1008. https://doi.org/10.21914/anziamj.v52i0.3963

[9] Keane, T. (2011) Combat Modelling with Partial Differential Equations. Applied Mathematical Modelling, 35, 2723-2735. https://doi.org/10.1016/j.apm.2010.11.057

[10] Pincombe, B.M. and Pincombe, A.H. (2016) Mass Action Models of Falklands War Battles. ANZIAM Journal, 57, 235-252. https://doi.org/10.21914/anziamj.v57i0.10450

[11] Pincombe, A.H., Pincombe, B.M. and Pearce, C.E.M. (2016) Dispersed Combat as Mass Action with Finite Search. ANZIAM Journal, 57, 305-319. https://doi.org/10.21914/anziamj.v57i0.10447

[12] Millikan, J., Wong, M. and Grieger, D. (2013) Suppression of Dismounted Soldiers: towards Improving Dynamic Threat Assessment in Closed Loop Combat Simulations. In: Piantadosi, J., Anderssen, R.S. and Boland, J., Eds., 20 th International Congress on Modelling and Simulation (MODSIM2013), Adelaide, 2013, 1054-1060.

[13] Bowden, F.D.J., Pincombe, B.M. and Williams, P.B. (2015) Feasible Scenario Spaces: A New Way of Measuring Capability Impacts. In: Weber, T., McPhee, M.J. and Anderssen, R.S., Eds, 21 st International Congress on Modelling and Simulation (MODSIM2015), Gold Coast, 2015, 836-842.

[14] Ramirez, M., Papasimeon, M., Benke, L., Lipovetzky, N., Miller, T. and Pearce, A.R. (2017) Real-Time UAV Maneuvering via Automated Planning in Simulations. 26th International Joint Conference on Artificial Intelligence (IJCAI-17), Melbourne, 19-25 August 2017, 5243-5245.

[15] Ramirez, M., Papasimeon, Lipovetzky, N., Benke, L., Miller, T., Pearce, A.R., Scala, E. and Zamani, M. (2018) Integrated Hybrid Planning and Programmed Control for Real-Time UAV Maneuvering. Proceeding 17 th International Conference on $\mathrm{Au}$ tonomous Agents and Multiagent Systems (AAMAS 2018), Stockholm, 10-15 July 2018, 1318-1326.

[16] Perla, P. and Lehoczky, J. (1977) A New Approach to the Analysis of Stochastic Lanchester Processes. I. Time Evolution. Technical Report No. 135, Department of Statistics, Carnegie-Mellon.

[17] Amacher, M. and Mandallaz, D. (1986) Stochastic Versions of Lanchester Equations in Wargaming. European Journal of Operational Research, 24, 41-45. https://doi.org/10.1016/0377-2217(86)90008-1

[18] Perry, N. (2009) Application of Black Scholes Complexity Concepts to Combat 
Modelling. DSTO-TR-2318.

[19] Perry, N. (2011) Applications of Historical Analyses in Combat Modelling. DSTO-TR-2643.

[20] Ivancevic, V. and Ivancevic, T. (2007) Neuro-Fuzzy Associative Machinery for Comprehensive Brain and Cognition Modelling. Springer, Berlin. https://doi.org/10.1007/978-3-540-48396-0

[21] Haken, H. (2002) Brain Dynamics, Synchronization and Activity Patterns in Pulse-Codupled Neural Nets with Delays and Noise. Springer, Berlin.

[22] Haken, H. (1993) Advanced Synergetics: Instability Hierarchies of Self-Organizing Systems and Devices. 3nd Edition, Springer, Berlin.

[23] Kawasaki, K. (1973) Simple Derivations of Generalized Linear and Nonlinear Langevin Equations. Journal of Physics A: Mathematical, Nuclear and General, 6, 1289. https://doi.org/10.1088/0305-4470/6/9/004

[24] Ichimaru, S. (1973) Basic Principles of Plasma Physics: A Statistical Approach. Benjamin/Cummings, Reading, MA.

[25] Janssen, H.K. (1976) On a Lagrangean for Classical Field Dynamics and Renormalization Group Calculations of Dynamical Critical Properties. Zeitschrift für Physik B Condensed Matter, B, 23, 377-380. 


\section{Appendix: Deterministic and Random Strikes}

In this section we develop the necessary physical background of both deterministic (Newtonian) and random (Langevin) strikes, in the following seven steps (compare with [21]):

Single strike. To start with, consider a one-dimensional (1D) damped motion of a ball of unit mass, moving with velocity $V$, within a viscous fluid with damping coefficient $\gamma$. The ball is pushed by an external striking force, defined in terms of the Dirac delta-function $\delta(t)$, with intensity/amplitude $\alpha$, occurring at time $t=\tau$, as:

$$
f_{\text {sng }}(t)=\alpha \delta(t-\tau) .
$$

From the second Newton's law we obtain the single-strike equation of motion:

$$
\dot{v}=-\gamma v(t)+\alpha \delta(t-\tau),
$$

where overdot represents the time derivative. The general solution of this ordinary differential equation (ODE) is given in terms of the Green function:

$$
v(t)=\alpha G(t-\tau), \quad \text { where } G(t-\tau)= \begin{cases}0 & \text { for } t<\tau \\ \mathrm{e}^{-\gamma(t-\tau)} & \text { for } t \geq \tau\end{cases}
$$

Multi-strike. Instead of a single strike given by the delta-force (9) we will now hit the ball by a series of $N$ strikes with intensities $\alpha_{j}$, occurring at a sequence of times $\left\{\tau_{j}\right\}$, so that the total striking force becomes:

$$
f_{\text {dis }}(t)=\sum_{j=1}^{N} \alpha_{j} \delta\left(t-\tau_{j}\right)
$$

If we replace this multi-strike force into the $\operatorname{ODE}(10)$, we obtain the multi-strike equation of motion:

$$
\dot{v}=-\gamma v(t)+\sum_{j=1}^{N} \alpha_{j} \delta\left(t-\tau_{j}\right) .
$$

The general solution of the $\mathrm{ODE}(12)$ is:

$$
v(t)=\sum_{j=1}^{N} \alpha_{j} G\left(t-\tau_{j}\right), \quad \text { where } G\left(t-\tau_{j}\right)= \begin{cases}0 & \text { for } t<\tau_{j} \\ \mathrm{e}^{-\gamma\left(t-\tau_{j}\right)} & \text { for } t \geq \tau_{j}\end{cases}
$$

Discrete and continuous striking spectra. Now, we can imagine that, within a certain time interval, the strikes are continuously exerted on the ball, in a rapid-fire fashion, so that the total striking force $f_{\text {tot }}(t)$ includes both the discrete spectrum $f_{\text {dis }}(t)$ and a continuous spectrum $f_{\text {con }}(t)$ :

$$
\begin{aligned}
f_{\text {tot }}(t) & =f_{\text {dis }}(t)+f_{\text {con }}(t) \\
& =\sum_{j=1}^{N} \alpha_{j} \delta\left(t-\tau_{j}\right)+\int_{t_{0}}^{t_{1}} \alpha(\tau) \delta(t-\tau) \mathrm{d} \tau \\
& \equiv \sum_{j=1}^{N} \alpha_{j} \delta\left(t-\tau_{j}\right)+\int_{t_{0}}^{t_{1}} f_{\text {con }}(t) \delta(t-\tau) \mathrm{d} t .
\end{aligned}
$$

In this way we obtain the more general multi--strike equation of motion: 


$$
\begin{aligned}
\dot{v} & =-\gamma v(t)+f_{\text {dis }}(t)+f_{\text {con }}(t) \\
& =-\gamma v(t)+\sum_{j=1}^{N} \alpha_{j} \delta\left(t-\tau_{j}\right)+\int_{t_{0}}^{t_{1}} \alpha(t) \delta(t-\tau) \mathrm{d} t \\
& \equiv-\gamma v(t)+\sum_{j=1}^{N} \alpha_{j} \delta\left(t-\tau_{j}\right)+\int_{t_{0}}^{t_{1}} f_{\text {con }}(t) \delta(t-\tau) \mathrm{d} t .
\end{aligned}
$$

The general solution of Equation (14) is:

$$
\begin{aligned}
v(t) & =\sum_{j=1}^{N} \alpha_{j} G\left(t-\tau_{j}\right)+\int_{t_{0}}^{t_{1}} f_{\text {con }}(t) G(t-\tau) \mathrm{d} t \\
& =\sum_{j=1}^{N} \alpha_{j} G\left(t-\tau_{j}\right)+\int_{t_{0}}^{t_{1}} f_{\text {con }}(t) \mathrm{e}^{-\gamma(t-\tau)} \mathrm{d} t,
\end{aligned}
$$

where

$$
G\left(t-\tau_{j}\right)= \begin{cases}0 & \text { for } t<\tau_{j} \\ \mathrm{e}^{-\gamma\left(t-\tau_{j}\right)} & \text { for } t \geq \tau_{j}\end{cases}
$$

Bidirectional random strikes. Now, we introduce random strikes, by denoting the times at which strikes occur by $\tau_{j}$ and indicate their 1D direction by $( \pm 1)_{j}$, where the choice of the plus or minus sign is random. Assuming (for simplicity) the same intensity $\alpha$ for all random strikes, the random striking force (with random numbers: $\rho \in[0,1]$ ) can be defined as:

$$
f_{\text {rnd }}(t)=\alpha \sum_{j=1}^{N} \delta\left(t-\tau_{j}\right)( \pm \rho)_{j} .
$$

Inclusion of the random striking force (16) into Equation (14) gives the randomized equation of motion:

$$
\begin{aligned}
\dot{v} & =-\gamma v(t)+f_{\text {dis }}(t)+f_{\text {con }}(t)+f_{\text {rnd }}(t) \\
& =-\gamma v(t)+\sum_{j=1}^{N} \alpha_{j} \delta\left(t-\tau_{j}\right)+\int_{t_{0}}^{t_{1}} \alpha(\tau) \delta(t-\tau) \mathrm{d} \tau+\alpha \sum_{j=1}^{N} \delta\left(t-\tau_{j}\right)( \pm \rho)_{j} .
\end{aligned}
$$

The general solution of Equation (17) is:

$$
v(t)=\sum_{j=1}^{N} \alpha_{j} G\left(t-\tau_{j}\right)+\int_{t_{0}}^{t_{1}} f(\tau) \mathrm{e}^{-\gamma(t-\tau)} \mathrm{d} \tau+\alpha \sum_{j=1}^{N} G\left(t-\tau_{j}\right)( \pm \rho)_{j}
$$

where

$$
G\left(t-\tau_{j}\right)= \begin{cases}0 & \text { for } t<\tau_{j} \\ \mathrm{e}^{-\gamma\left(t-\tau_{j}\right)} & \text { for } t \geq \tau_{j}\end{cases}
$$

Average random strikes. If we now observe many similar scenarios, we can perform an average $\langle\ldots\rangle$ over all of them, to obtain the average random striking force:

$$
\left\langle f_{\text {rnd }}(t)\right\rangle=\alpha\left\langle\sum_{j=1}^{N} \delta\left(t-\tau_{j}\right)( \pm \rho)_{j}\right\rangle .
$$

Assuming that the times $\tau_{j}$ at which the strikes happen are independent of 
the direction $( \pm 1)_{j}$ of the strikes, we can split (18) into the product:

$$
\left\langle f_{\text {rnd }}(t)\right\rangle=\alpha\left\langle\sum_{j=1}^{N} \delta\left(t-\tau_{j}\right)\right\rangle\left\langle( \pm \rho)_{j}\right\rangle .
$$

Next, if the strikes happen in both directions with equal frequency, they simply cancell each other:

$$
\left\langle( \pm 1)_{j}\right\rangle=0 \Rightarrow\left\langle f_{\text {rnd }}(t)\right\rangle=0 .
$$

In order to characterize the strength of the force (16), consider a quadratic expression in $f$ by calculating the correlation function for two times $t, t^{\prime}$ (see [22]):

$$
\left\langle f_{\text {rnd }}(t) f_{\text {rnd }}\left(t^{\prime}\right)\right\rangle=\alpha^{2}\left\langle\sum_{j} \delta\left(t-\tau_{j}\right)( \pm \rho)_{j} \sum_{k} \delta\left(t^{\prime}-t_{k}\right)( \pm \rho)_{k}\right\rangle,
$$

which simplifies into a single sum as:

$$
\left\langle f_{\text {rnd }}(t) f_{\text {rnd }}\left(t^{\prime}\right)\right\rangle=\alpha^{2}\left\langle\sum_{j} \delta\left(t-\tau_{j}\right) \delta\left(t^{\prime}-t_{k}\right)\right\rangle,
$$

since the ones for $j=k$ become 1 and for $j \neq k$ cancel each other. This correlation function is usually evaluated by assuming the Poisson process for the times of the strikes.

Simple Langevin equation. Now, if we assume: 1) that the average random striking force (18) vanishes, and 2) that the correlation function (19) can be further simplified as:

$$
\left\langle f_{\text {rnd }}(t) f_{\text {rnd }}\left(t^{\prime}\right)\right\rangle=q \delta\left(t-t_{0}\right),
$$

where $t_{0}$ denotes the mean time between any two strikes and $q=\alpha^{2} / t_{0}$ represents the random fluctuation, the equation of motion driven solely by the random striking force $f_{\text {rnd }}(t)$ becomes the simple Langevin equation:

$$
\dot{v}=-\gamma v(t)+f_{\text {rnd }}(t) \text {. }
$$

Since both directions are equally possible, they cancel each other, and the average velocity vanishes. Therefore, from the integral solution (15) of Equation (14) we obtain

$$
\left\langle v(t) v\left(t^{\prime}\right)\right\rangle=\left\langle\int_{t_{0}}^{t} \mathrm{~d} \tau \int_{t_{0}}^{t^{\prime}} f_{\text {rnd }}(\tau) f_{\text {rnd }}\left(\tau^{\prime}\right) \mathrm{e}^{-\gamma(t-\tau)} \mathrm{e}^{-\gamma\left(t^{\prime}-\tau^{\prime}\right)} \mathrm{d} \tau^{\prime}\right\rangle .
$$

In the steady--state, Equation (22) reduces to:

$$
\left\langle v(t) v\left(t^{\prime}\right)\right\rangle=\frac{q}{2 \gamma} \mathrm{e}^{-\gamma(t-\tau)},
$$

which, for equal times $t$ and $\tau$, simplifies into:

$$
\left\langle v(t)^{2}\right\rangle=\frac{q}{2 \gamma} .
$$

Hamilton-Langevin equation. The simple 1D Langevin Equation (21) can be generalized in the framework of Hamiltonian dynamics (see [23]) to the 
following $n \mathrm{D}$ form:

$$
\dot{v}_{i}=k_{B} T \sum_{j=1}^{n}\left[v_{i}, v_{j}\right] \frac{\partial H}{\partial v_{j}}-\sum_{j=1}^{n} \gamma_{i j} \frac{\partial H}{\partial v_{j}}+f_{i}^{\mathrm{rnd}}(t),
$$

where $k_{B}$ and $T$ denote the Boltzmann constant and the temperature, $\left[v_{i}, v_{j}\right]$ represents the Poisson bracket and $H$ is the Hamiltonian of the system given as $H=-\ln \left(p_{0}\right)$, where $p_{0}\left(v_{i}\right)$ is the equilibrium probability distribution of $v_{i}$. The Gaussian-distributed random forces $f_{i}^{\text {rnd }}(t)$ have the correlation function:

$$
\left\langle f_{i}^{\mathrm{rnd}}(t) f_{j}^{\mathrm{rnd}}\left(t^{\prime}\right)\right\rangle=2 \gamma_{i j} \delta\left(t-t^{\prime}\right),
$$

which implies the Onsager reciprocity relations for the damping coefficients $\gamma_{i j}=\gamma_{j i}$.

Probabilistic interpretations. The Hamilton-Langevin Equation (23) has two probabilistic interpretations (see [24] [25]):

\section{- Fokker-Planck equation:}

$$
\frac{\partial P(v, t)}{\partial t}=\sum_{i, j} \frac{\partial}{\partial v_{i}}\left(-k_{B} T\left[v_{i}, v_{j}\right] \frac{\partial H}{\partial v_{j}}+\gamma_{i j} \frac{\partial H}{\partial v_{j}}\right) P(v, t),
$$

with a stationary solution given by the equilibrium distribution: $P(v, t)=p_{0}\left(v_{i}\right)=c \mathrm{e}^{-H}$, with some constant $c$, and

\section{- Feynman path integral:}

$\int \mathrm{d} v \mathrm{~d} \tilde{v} P(v, \tilde{v})=N \int \mathrm{d} v \mathrm{~d} \tilde{v} \exp [L(v, \tilde{v})]$, with

$$
L(v, \tilde{v})=\int \mathrm{d} t \sum_{i, j}\left[\tilde{v}_{i} \gamma_{i j} \tilde{v}_{j}-\tilde{v}_{i}\left(\delta_{i j} \dot{v}_{j}-k_{B} T\left[v_{i}, v_{j}\right] \frac{\partial H}{\partial v_{j}}+\gamma_{i j} \frac{\partial H}{\partial v_{j}}\right)\right],
$$

where $\tilde{v}_{i}$ represent the auxiliary-response variables and $N$ is the normalization constant. 